\title{
MERCADEO EN LAS EMPRESAS DE CALZADO DE CÚCUTA EN EL MARCO DE LA RESPONSABILIDAD SOCIAL.
}

\section{MARKETING IN THE CÚCUTA FOOTWEAR COMPANIES IN THE FRAMEWORK OF SOCIAL RESPONSIBILITY.}

\section{Byron Medina Delgado'}

Wlamyr Palacios Alvarado²

\section{Luis Leonardo Camargo Ariza ${ }^{3}$}

UFPS- Universidad del Magdalena

\section{RESUMEN}

El enfoque de mercadeo social en la actualidad adquiere un papel preponderante que armoniza con los programas de responsabilidad social empresarial y que las organizaciones deben implementar para atender los problemas sociales, como son la satisfacción de las necesidades en las clases sociales de bajos recursos. El objetivo

1 Universidad Francisco de Paula Santander,

Cúcuta, Colombia. Docente - Investigador

Orcid: $h$ ttps://orcid.org/0000-0003-0754-8629

byronmedina@ufps.edu.co

2 Universidad Francisco de Paula Santander,

Cúcuta, Colombia. Docente - Investigador

Orcid: https://orcid.org/0000-0002-4292-4178

wlamyrpalacios@ufps.edu.co

3 Universidad del Magdalena, Santa Marta, Colombia. Docente - Investigador

Orcid: https://orcid.org/0000-0002-7956-441X

Icamargoa@unimagdalena.edu.co del artículo es entender la implementación del mercadeo social y la responsabilidad social empresarial en las fábricas de calzado de Cúcuta. El trabajo aplica metodología cualitativa y cuantitativa a través de investigación documental y descriptiva mediante encuestas aplicadas a 294 gerentes de las empresas. Los resultados indican que las fábricas de calzado de Cúcuta presentan una cobertura del mercado internacional del $7 \%$, orientación del mercadeo a las clases sociales altas del $69 \%$ y programas de responsabilidad social enfocados en los incentivos al recurso humano con el $70 \%$. Se concluye que las empresas deben mejorar la integración con mercados globales, redistribuir la orientación del mercadeo con respecto a las clases sociales e incrementar los programas externos de responsabilidad social. 


\section{PALABRAS CLAVE}

Clases sociales, cobertura de mercados, mercadeo social, responsabilidad social empresarial

\section{ABSTRACT}

The social marketing approach at present acquires a preponderant role that harmonizes with corporate social responsibility programs and that organizations must implement to address social problems, such as meeting the needs of lowincome social classes. The objective of the article is to understand the implementation of social marketing and corporate social responsibility in the shoe factories of Cúcuta. The work applies qualitative and quantitative methodology through documentary and descriptive research through surveys applied to 294 company managers. The results indicate that the shoe factories of Cúcuta present a $7 \%$ international market coverage, a $69 \%$ marketing orientation to upper social classes, and social responsibility programs focused on human resource incentives with 70 $\%$. It is concluded that companies should improve integration with global markets, redistribute the orientation of marketing with respect to social classes and increase external programs of social responsibility.

\section{KEY WORDS}

Social classes, market coverage, social marketing, corporate social responsibility

\section{INTRODUCCIÓN}

Los países en vía de desarrollo atraviesan dificultades económicas y sociales como bajo poder adquisitivo y altas tasas de desempleo, como es el caso de Colombia y en especial la ciudad de Cúcuta, que por su ubicación en zona de frontera está afectada por los cambios ideológicos de Venezuela y como tal requiere de opciones que apunten a la optimización de procesos y acciones orientadas a la satisfacción de necesidades de parte de los diversos sectores, con base en el mercadeo social que surge como una solución a las clases sociales de bajos recursos.

Ante estas precisiones, el sector del calzado de Cúcuta debe afrontar el desafío de implementar un mercadeo con responsabilidad social, mediante la definición de estrategias que contribuyan con los cambios en la gestión gerencial, en materia de mercedo social que beneficien a la población de bajos recursos.

En este sentido, el presente artículo tiene como objetivo entender cómo el mercadeo social y la responsabilidad social empresarial (RSE) son implementados en las fábricas de calzado de Cúcuta; su desarrollo se basa en la metodología cualitativa y cuantitativa que aplica investigación documental e investigación descriptiva mediante la aplicación de una encuesta a una muestra de fábricas de calzado de Cúcuta. En la segunda sección aparece el marco teórico que incluye el mercadeo social y la RSE. En la tercera sección se analiza la metodología aplicada en cuanto al tipo de investigación y la población y muestra. En la cuarta sección se presentan los resultados de las encuestas, los cuales señalan que las fábricas de calzado en Cúcuta tienen una mayor cobertura de mercado local, el mercadeo está orientado con mayor énfasis a las clases sociales altas y muy altas y en cuanto a la RSE son más endógenas que exógenas. Finalmente, en la quinta sección se encuentran las conclusiones, resaltando principalmente que las empresas del calzado de Cúcuta están obligadas a realizar una revisión permanente de sus modelos de negocios teniendo como referencia nuevos nichos de mercado y el mayor crecimiento poblacional en la base de la pirámide. 


\section{MARCO TEÓRICO}

\subsection{Mercadeo social}

La Asociación de Marketing de Estados Unidos (American Marketing Association, AMA), citado en Álvarez (2007) el Marketing es "el proceso de planear y ejecutar la concepción, precio, promoción y distribución de ideas, bienes y servicios para crear intercambios que puedan satisfacer las necesidades de los consumidores y los objetivos de la organización" (Alvarez, 2007, p.67); otro autor como Stanton señala que "el marketing consiste en actividades ideadas para generar y facilitar intercambios con la intención de satisfacer necesidades, deseos humanos o de organizaciones" (Álvarez, 2007, p.67).

En esta materia, el mercadeo social implica una gestión orientada hacia la atención de necesidades en la base de la pirámide con responsabilidad social, que identifique las causas sociales de una comunidad y desarrolle estrategias para divulgar las acciones sociales por parte de organizaciones preocupadas por el bienestar social, conforme lo señalan Giuliani, Monteiro, Zambon, Betanho y Lima (2012).

Dicho de otro modo y en atención con lo expuesto, las compañías tendrían que dejar de competir entre sí, de acuerdo con Chan y Mauborgne (2005), escenario en donde la competencia pierde toda importancia, dado que se identifican espacios desconocidos en el mercado, llamados por los autores mencionados como los océanos azules a donde se apuntan las estrategias de mercadeo para optimizar oportunidades y minimizar riesgos, escenario que obliga a seleccionar una posición estratégica centrada en bajos costos y diferenciación mediante la innovación.

Los escenarios anteriores, difieren de los que comúnmente son atendidos por empresas cuyo mercadeo es orientado a la parte más pequeña y acaudala, la punta de la pirámide, según Mutis y Ricart (2008) olvidándose de la base de la pirámide en donde aparecen una gran cantidad de compradores.

Conforme con Chan y Mauborgne (2005), la estructura del mercado está determinada por la oferta y la demanda que tiene directa influencia en el comportamiento de vendedores y compradores y, en otros factores externos a la estructura del mercado como son los cambios en las condiciones económicas y los avances tecnológicos, cambios que son previstos por empresarios que poseen una actitud proactiva basada en la innovación para generar valor agregado al cliente.

Finalmente, debe señalarse, que el mercadeo social está generando impactos positivos en las organizaciones y en el entorno socio-económico, tales impactos de acuerdo con Aristizábal, León y Arias (2007) son los que se evidencian en el apoyo social que desarrollan las empresas en las comunidades, logrando procesos de apalancamiento organizacional en la comunidad; también en cuanto a la transformación de la relación organización-comunidad, y a la transformación y posicionamiento de la imagen organizacional.

\subsection{Responsabilidad social empresarial}

El secretario general de las Naciones Unidas en el foro de Davos de 1999 exhorta a interrelacionar el poder de los mercados con los valores universales como forma de extender los beneficios de la globalización; allí se origina el llamado pacto global que promueve el compromiso público de las empresas en torno a nueve principios sobre derechos humanos, laborales y medioambientales, según Gilli (2006).

Además, aparece otro antecedente conocido como el libro Verde la Comunidad Europea en el 2002, el cual promueve la responsabilidad empresarial en sus dimensiones interna $y$ 
externa, dada la incapacidad del estado para resolver los problemas sociales, situación que resulta especialmente marcada en ciertas regiones, por ejemplo en Latinoamérica, donde los fondos públicos se invierten electoralmente en lugar de promover el desarrollo humano; la pobreza pasa a ser un recurso de la política, de acuerdo con Gilli (2006).

Dentro de este marco y frente a la necesidad de responder a requerimientos de la sociedad, surge la RSE que resulta de acciones voluntarias que contribuyan al desarrollo, crecimiento y mejoramiento de la calidad de la vida de un grupo social específico, para así, reflejar las consecuencias de sus actos, en actitudes y resultados positivos, conforme lo señalan Duque, Cardona y Rendón (2013).

La RSE de acuerdo con Bowen (1953), se refiere a los compromisos de los empresarios para aplicar políticas, tomar decisiones o seguir las líneas de acción que son deseables en términos de los objetivos y valores de la sociedad, es decir, el empresario debe actuar para servir a la sociedad en lugar de tener como objetivo la maximización de los beneficios como único fin de la empresa.

Actualmente, la RSE es considerada como un enfoque de gestión estratégico generador de oportunidades, innovación y ventaja competitiva para las organizaciones, con una repercusión en su imagen y reputación, según Porter y Kramer, (2002), citados en Peláez-León y GarcíaSolarte (2014); ante esta perspectiva, la RSE se convierte en una estrategia que apunta a la satisfacción de las necesidades y expectativas de sus grupos de interés. Cabe señalar que la empresa para suministrar bienes y servicios a un mercado, necesita emplear personas y recursos y operar en un medio, función que es responsabilidad de los directivos y que en el mejor de los casos malgastan recursos $y$, por lo tanto, nada agregan al valor del producto o a la satisfacción del cliente, en otros casos se presentan maltrato al personal, falta de transparencia en la relación con proveedores, publicidad engañosa, mala calidad del producto o contaminación ambiental, entre otros, lo cual afecta a la imagen de la empresa, conforme a Gilli (2006).

A título ilustrado, merece la pena destacar que las empresas deben medir el cumplimiento de los compromisos sociales utilizando como herramienta el balance social, también es conocido como estado de responsabilidad social, según Chapman (1988), citado en Oliveros (2016), documento que presenta indicadores de responsabilidad social según Gilli (2011), que aparece en Oliveros (2016).

Finalmente, el balance social se define como el estado que permite conocer de manera sistemática las acciones de responsabilidad social de la organización, midiéndola cualitativa y cuantitativamente de forma permanente y sistemática, para identificar los programas que favorezcan el desarrollo efectivo de la acción social e informar a los agentes internos y externos, sobre el desempeño social de la organización durante un período determinado, conforme lo señala Oliveros (2016).

\section{METODOLOGÍA}

\subsection{Tipo de investigación}

La investigación documental es definida como "el estudio de problemas con el propósito de ampliar y profundizar el conocimiento de su naturaleza, con apoyo, principalmente, en trabajos previos" (Universidad Pedagógica Experimental Libertador, 2002, p.28). En este estudio las fuentes secundarias de información como primer nivel del conocimiento, contenidas en artículos, proyectos, documentos, escritos y publicaciones, entre otras, relacionadas con los temas del mercadeo y la responsabilidad social de las fábricas de calzado de Cúcuta, permitieron un análisis contenido en el marco 
teórico del presente artículo, mediante la recolección de datos utilizando instrumentos, como hojas de análisis, que facilitaron la revisión y organización de la literatura según bibliografía citada y referenciada.

Además, con el propósito de profundizar en un segundo nivel de conocimiento se aplica una investigación descriptiva con el objeto de "establecer comportamientos concretos y a su vez descubrir y comprobar la posible asociación de las variables de investigación" (Méndez, 2007, p.231), con el propósito de determinar los alcances de mercado de las fábricas de calzado de Cúcuta en materia de cobertura y clases sociales a que están orientadas las acciones de mercadeo.

El método aplicado es de tipo cualitativo y cuantitativo, en otras palabras, es mixto con criterio deductivo.

\subsection{Población y muestra}

La población objeto de estudio está identificada como las fábricas de calzado de Cúcuta que alcanzan un total de 1.251 empresas, de acuerdo con el portal de la Cámara de Comercio de Cúcuta, Compite Reporte Empresarial (2021), población determinada que se analiza a través de una muestra representativa, mediante muestreo probabilístico aleatorio simple; muestra calculada de acuerdo con la siguiente expresión estadística.

$$
n=\frac{N Z^{2} P Q}{N e^{2}+Z^{2} P Q}
$$

En donde:

$n=$ Tamaño de la muestra.

$N=$ Tamaño de la población igual a 1.251

fábricas de calzado en Cúcuta.

$Z=$ Nivel de confianza del $95 \%$, equivalente a 1,96 .
$P=$ Probabilidad de mercadeo social igual al 50 $\%$.

$Q=$ Probabilidad de falta de orientación hacia el mercadeo social igual al $50 \%$.

$e=$ Margen de error permisible igual al $5 \%$.

Reemplazando las variables se obtienen 294 fábricas de calzado de Cúcuta.

$$
n=\frac{1.251 \times(1,96)^{2} \times 0,5 \times 0,5}{1.251 x(0,05)^{2}+(1,96)^{2} \times 0,5 \times 0,5}=294
$$

\subsection{Instrumentos de recolección de información}

\section{FUENTES PRIMARIAS}

Identificadas como información que suministra la muestra de fabricantes de calzado de Cúcuta, recolectada a través del cuestionario para determinar cobertura de mercados $y$ responsabilidad social.

\section{FUENTES SECUNDARIAS}

Identificadas como fuentes de información contenidas en artículos, libros, documentos, estudios y proyectos de grado, relacionadas con el mercadeo y la responsabilidad social empresarial, que se estudian utilizando hojas de análisis como instrumento.

\section{RESULTADOS}

Con el propósito de determinar los alcances de mercado de las fábricas de calzado de Cúcuta en materia de cobertura y clases sociales a que están orientadas las acciones de mercadeo, se aplicó cuestionario a una muestra de 294 gerentes de estas empresas, cuyos resultados se presentan en las tablas 1,2 y 3 . 
Tabla 1. Cobertura de mercados.

\begin{tabular}{|c|c|c|}
\hline Categorías & Frecuencia & $\%$ \\
\hline Local & 73 & 25 \\
\hline Regional & 84 & 28 \\
\hline Nacional & 117 & 40 \\
\hline Internacional & 20 & 7 \\
\hline Total & $\mathbf{2 9 4}$ & $\mathbf{1 0 0}$ \\
\hline
\end{tabular}

Fuente: Cuestionario.

Los gerentes de las 294 fábricas de calzado de Cúcuta, señalan que la cobertura de mercado es local de acuerdo con el $25 \%$, regional el $28 \%$, nacional el $40 \%$ e internacional el $7 \%$; es decir, el resultado indica una baja integración con los mercados globales, teniendo en cuenta la baja participación en mercados internacionales frente a la cobertura local, regional y nacional.

Tabla 2. Clases sociales a las que se orienta el mercadeo de la fábrica.

\begin{tabular}{|c|c|c|}
\hline Categorías & Frecuencia & $\%$ \\
\hline Baja & 3 & 1 \\
\hline Media & 87 & 30 \\
\hline Alta & 148 & 50 \\
\hline Muy alta & 56 & 19 \\
\hline Total & $\mathbf{2 9 4}$ & $\mathbf{1 0 0}$ \\
\hline
\end{tabular}

Fuente: Cuestionario.

Los gerentes de las fábricas de calzado de Cúcuta señalan que el mercadeo está orientado a las clases sociales bajas según el $1 \%$, media el $30 \%$, altas el $50 \%$ y muy altas el $19 \%$, resultados que son un indicio de que el interés se centra en mayor proporción, con $69 \%$, en las clases altas y muy altas.

Tabla 3. Programas de responsabilidad social.

\begin{tabular}{|c|c|c|}
\hline Categorías & Frecuencia & $\%$ \\
\hline Incentivos al recurso humano & 205 & 70 \\
\hline Integración con la familia & 64 & 21 \\
\hline Integración con la comunidad & 25 & 9 \\
\hline Total & $\mathbf{2 9 4}$ & $\mathbf{1 0 0}$ \\
\hline
\end{tabular}

Fuente: Cuestionario.

Los gerentes de las fábricas de calzado de Cúcuta señalan que los programas de responsabilidad social están orientados hacia incentivos al recurso humano según el $70 \%$, integración con las familias de los empleados el $21 \%$, e integración con la comunidad el $9 \%$, resultados que evidencian un bajo énfasis hacia los programas externos de responsabilidad social; las fábricas son más endógenas que exógenas. 


\section{CONCLUSIONES}

Las empresas de calzado de Cúcuta están obligadas a realizar una revisión permanente de sus modelos de negocios teniendo como referencia nuevos nichos de mercado y el mayor crecimiento poblacional en la base de la pirámide.

Los océanos azules como estrategia apuntan hacia segmentos de mercado caracterizados por una población de bajos ingresos, que presenta necesidades básicas insatisfechas, en donde la competencia es irrelevante.

Es prioritario que las empresas de calzado de Cúcuta asuman la responsabilidad por los problemas sociales tanto internos como externos y que contribuyan a su solución, en especial en la base de la pirámide.

Las fábricas de calzado de Cúcuta presentan deficiente cobertura del mercado internacional, orientación del mercadeo a las clases sociales altas y programas de responsabilidad social enfocados en los incentivos al recurso humano. Esto implica mejorar la integración con mercados globales, redistribuir la orientación del mercadeo con respecto a las clases sociales e incrementar los programas externos de responsabilidad social.

\section{REFERENCIAS}

Alvarez Pinto, F. D. (2007). Planificación Estrategica De Marketing. Perspectivas, (20), 67-104.

Aristizábal, Botero, Carlos Andrés, León Restrepo, Mario, Arias Pérea, José, (2007), Impactos del mercadeo social en organizaciones de servicios ${ }^{*}$ Semestre Económico, vol.10 no.19 Universidad Luis Amigó., Medellín.
Bowen, H. (1953). Social responsibilities of the businessman. New York, Estados Unidos: Harper \& Row.

Chan Kim, W., y Mauborgne, R. (2005). La estrategia del océano azul. In Harvard Business Review (Vol. 82). Retrieved from https://genniux.net/wiki/elabs/pub2/Trash/ EmpowerLabsIntraEstrategiaBlueOcean/La_ estrategia_del_oceano_azul.pdf

Duque Orozco, Yenni V.; Cardona Acevedo, Marleny; Rendón Acevedo, Jaime A., (2013), Responsabilidad Social Empresarial: Teorías, índices, estándares y certificaciones, Cuadernos de Administración, vol. 29, núm. 50, julio-diciembre, pp. 196-206 Universidad del Valle Cali, Colombia.

Gilli, Juan José, (2006), Responsabilidad Social Revista Científica "Visión de Futuro", vol. 5, núm. 1, Universidad Nacional de Misiones, Argentina.

Giuliani, Antonio Carlos; Monteiro, Thel Augusto; Zambon, Marcelo Socorro; Betanho, Cristiane; Lima Faria, Luiz Henrique, (2012), El Marketing Social, el Marketing relacionado con causas sociales y la Responsabilidad Social Empresarial, Caso del Supermercado Pão De Açúcar, de Brasil Invenio, vol. 15, núm. 29, Universidad del Centro Educativo Latinoamericano Rosario, Argentina, pp. 1127.

Méndez, Carlos, (2007), Metodología, Diseño y desarrollo del proceso de investigación con énfasis en ciencias empresariales, $4^{\circ}$ Edición, Editorial Limusa, Bogotá D.C.

Mutis, J., y Ricart, J. E. (2008). Innovación en modelos de negocio: La Base de la Pirámide como campo de experimentación. Universia Business Review, (18), 10-27. Disponible en https://www.redalyc.org/pdf/433/ Resumenes/Resumen_43301802_1.pdf 
Oliveros Villegas Miguel Ángel, (2016), El balance Social como Herramienta de Responsabilidad Social Empresarial: Una Aproximación Teórica, Sapienza Organizacional, vol. 3, núm. 6, pp. 93-106, Universidad de los Andes.

Peláez-León, Juan David; García-Solarte, Mónica, (2014), Responsabilidad social empresarial y gestión humana: una relación estratégica aplicada desde un modelo explicativo Entramado, vol. 10, núm. 2, pp. 90-111 Universidad Libre Cali, Colombia.

Universidad Pedagógica Experimental Libertador, (2002), Manual de Trabajos de Grado de Especialización y Maestría y Tesis Doctorales, Caracas. 